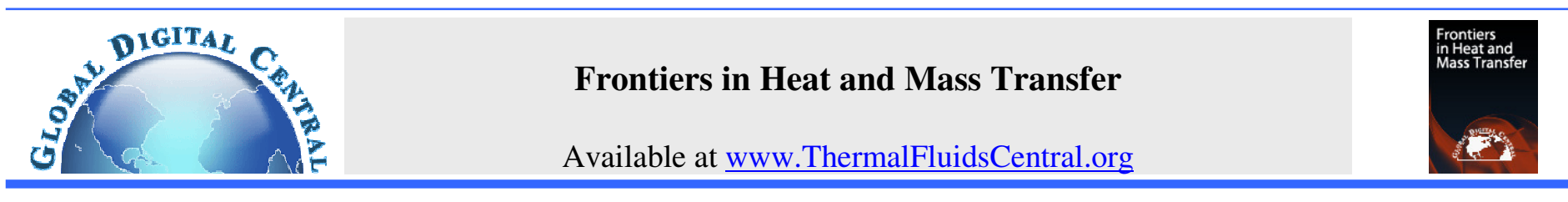

\title{
INVESTIGATION OF EFFECTIVE PARAMETERS ON ENTROPY GENERATION IN A SQUARE ELECTRONIC PACKAGE
}

\author{
Saeed Zaidabadi Nezad, Mohammad Mehdi Keshtkar ${ }^{*}$ \\ Mechanical Engineering Department, Kerman Branch, Islamic Azad University, Kerman, Iran.
}

\begin{abstract}
This work reports numerical optimization of natural convection in a square enclosure with local heating of the floor and symmetrical cooling of the sides. This analysis was done to optimize heat transfer from two semiconductors in a square electronic package. It became clear that the major part of entropy generation was due to irreversibility of heat transfer and a small amount of it was related to fluid friction. For heaters with equal power ratio, it is very unpleasant to place heaters near the middle plate of the enclosure, since peak temperatures and entropy generation are maximized.
\end{abstract}

Keywords: Natural convection, Optimization, Electronic package, Entropy generation, Power ratio.

\section{INTRODUCTION}

Heat transfer problems are the most important and practical problems in engineering sciences. Natural convection in enclosures and in the presence of heat-producing internal masses with different geometries is one of the problems recently raised. Based on articles published in this field, it can be claimed that this was associated with numerous numerical and hardware improvements in the last two decades. This focus is due to various applications of natural convection in various research and industrial fields such as cooling of circuits and electronic equipment, solar collectors, heat insulation, nuclear reactors, heat recovery systems, thermal design of buildings, air conditioning in environments, heat exchangers, double glazed windows, energy storage devices, etc. In these problems, there are various physical and computational challenges between fluid flow, heat transfer and boundary layer. This type of heat transfer can be divided into two groups of horizontal and vertical enclosures based on arrangement of the enclosure. Given the temperature limits of the components in the electronic packages, and considering that analysis of engineering heat transfer systems have many limitations based on the first law of thermodynamics, it is required to use the second law of thermodynamics in order to provide highly reliable results, as well as high-quality heat transfer.

Regarding the relationship between this problem and natural convection, this study explained natural convection theory in enclosures along with thermodynamic optimization of these problems. De Vahl Davis (1983) and Saitoh and Hirose (1989), carried out the early precise works on natural convection in enclosures. They numerically solved free convection in a square enclosure with insulated horizontal walls and vertical walls at two constant and different temperatures. Yong and Jang (2000) numerically analyzed natural convection in a squareshaped three-dimensional enclosure with a cubic mass producing heat in the center of the enclosure. They found that fluid flow inside the enclosure results from presence of two temperature differences; one is the difference in temperature between cold and hot walls on sides of the enclosure and the other is the difference in temperature produced by heat generator. Deng et al. (2002) studied free convection in a rectangular enclosure with separate heat sources. They showed that, in general, the role of constant temperature heat sources is higher than constant flux sources. Nithyadevi et al. (2007) examined natural convection in a rectangular enclosure with a hot side wall for nine different positions of thermal sources. In this study, the hot zone was located at the top, middle, and bottom of the enclosure and position of the cold zone varied from bottom to top of the enclosure to determine maximum and minimum heat transfer value. In another study of natural convection in an enclosure with a separate thermal source by Chadwick (2008), the results showed that maximum heat transfer rate is obtained in a high range of Grashof number when a heat source is used at the bottom of the enclosure. Enhancing combined natural convection in a rectangular enclosure with local heating from the below investigated by Onyango (2008), showed that the effect of change in dimensionless Reynolds, Prantel, Froude and Eckert numbers on flow and temperature field. Keshtkar and Gandjalikhan Nassab (2009) carried out a theoretical study to investigate the heat transfer characteristics of porous radiant burners.

At the another work by Keshtkar et al. (2009) studied the heat transfer characteristics of a new type of porous radiant air heater (PRAH) that operates on the basis of effective energy conversion method between flowing gas enthalpy and thermal radiation. A numerical study of combined natural convection in a rectangular enclosure with several separate thermal sources carried out by Gdhaidh (2014). This study evaluated the effect of Rayleigh number on flow and temperature field in different geometric conditions for air and fc-77. Ganjalikhan Nassab et al. (2014) investigated laminar forced convection flow over a backward-facing step in a duct. Keshtkar (2016) investigated fluid flow between two parallel flat plates that, partially filled with two-dimension porous media is investigated numerically using single relaxation time lattice Boltzmann method at pore scale. Rahimi et al. (2017) analyzed the natural convection heat transfer in nanofluid-filled cavity with partially heated and cooled walls included by internal heaters by different approaches such as heatline visualization, total and local entropy generation, average ad local Nusselt number using lattice Boltzmann numerical method. Salari et al. (2017) investigated numerical simulation for natural convection of two immiscible gas/liquid fluids, air and $\mathrm{Al} 2 \mathrm{O} 3$-water nanofluid, within a

* Corresponding author. Email: mkeshtkar54@yahoo.com, 
rectangular enclosure. Three-dimensional natural convection in a cuboid enclosure filled with DWCNTs-water nanofluid was studied by Rahimi et al. (2017). The heat transfer performance due to convective flow of nanofluid inside the enclosure at different temperature differences between side hot and cold walls were analyzed experimentally and numerically. The three-dimensional natural convection within the enclosures filled with two stratified and immiscible fluids of a nanofluid and air investigated by Salari et al. (2017). The lattice Boltzmann method (LBM) and discrete ordinates method (DOM) were applied to investigate the heat transfer in a square radiative-conductive media with heat flux and temperature boundary conditions by Keshtkar and Amiri (2017). Ezzatabadipour et al. (2017) investigated fluid flow through an isotropic porous medium with randomly arranged elliptical particles by the lattice Boltzmann method. Keshtkar and Ghazanfari (2017) studied the fluid flow and heat transfer analysis a two-dimensional square enclosure containing three hot obstacles affected by gravity and magnetic force placed on a ramp using Boltzmann method (LBM) applying multiple relaxation times (MRT).

At the present work, for numerical simulation of the problem, finite volume method (FVM), Power-Law approximation, staggered network, and SIMPLER algorithm were explained. Once the problem was numerically simulated by MATLAB, the results were presented as diagrams of streamlines, isotherms, heater surface temperatures, entropy generation rate caused by fluid friction and heat transfer in different states. This study tended to:

-evaluate the effect of physical and geometric factors on convection. -optimize temperature of heaters by minimizing their surface temperature in order to avoid hot spots in the enclosure.

-optimize the problem thermodynamically by minimizing the entropy generated in the system for heat transfer at the highest quality and uniform temperature distribution in the enclosure.

\section{MATERIALS AND METHODES}

This study evaluated entropy generation caused by natural convection in an enclosure which is heated locally from below by two constant flux heaters. This analysis is done to optimize heat transfer from two semiconductors in a square electronic package. In this simulation, electronic components are modeled in the form of constant flux heat sources and side walls in the form of isothermal wells. Flow and temperature fields are derived from numerical simulation of the equations of mass, momentum and energy conservation. Although dimensions of the enclosure and value of the thermal energy applied to it, are constant. Effect of dimensionless Rayleigh parameters, the ratio of length and power of heat sources, as well as their location on flow field, temperature, and entropy generation are evaluated.

\subsection{Geometry and Boundary Conditions}

Geometry of the problem and details of boundary conditions are shown in Figure 1, a square enclosure with a side length of $15 \mathrm{~cm}$ including two heat sources in the lower wall. Length of the left heater is $L_{h}$ and length of the right heater is $\varepsilon_{r} L_{h}$. The distance from the center to the center of heaters is shown as $X_{C}$. The fluid in the enclosure is air with $\mathrm{Pr}=0.7$. All enclosure walls are fixed. Temperature of the left and right walls is temperature of the cold wall $\left(T_{C}\right)$. Temperature of the ambient air is assumed to be $T_{\infty}=300 \mathrm{~K}$. The upper wall and the nonpyrogenic part of the lower wall are insulated. In solving the governing equations, the following boundary conditions are used:

Non-slip and non-permeability boundary condition considered for all walls $(U=V=0)$. Thermal boundary condition $\theta=0$ is for cold side walls. Thermal boundary condition $\partial \theta / \partial \mathrm{Y}=0$ is for the upper wall and the non-pyrogenic part of the lower wall.

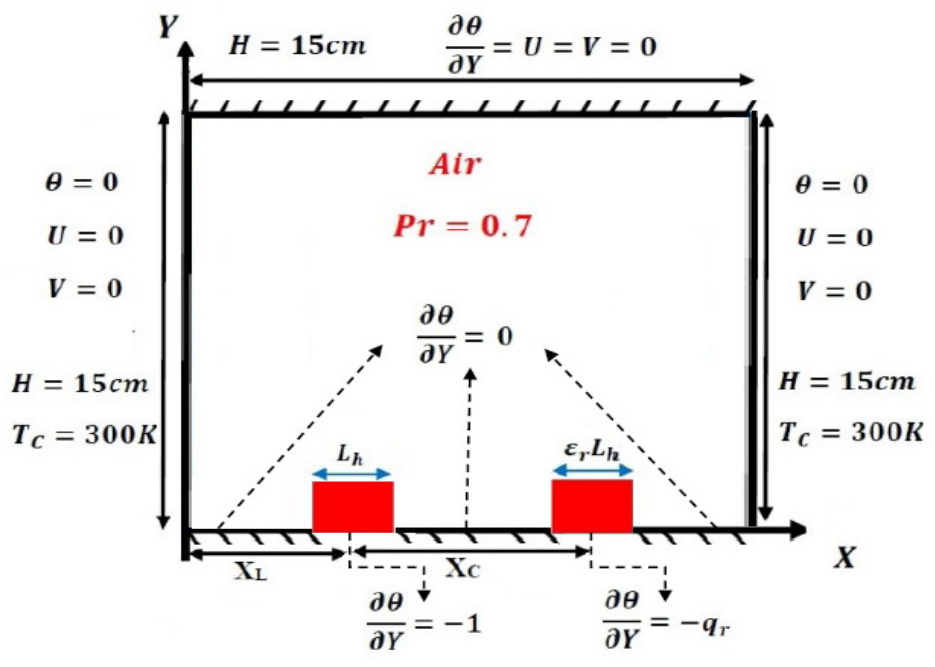

Fig. 1 Geometry of the problem with boundary conditions

For the left and right heaters, thermal boundary conditions are $\partial \theta / \partial \mathrm{Y}=-1$ and $\partial \theta / \partial \mathrm{Y}=-\mathrm{q}_{\mathrm{r}}$, respectively. In this study, fluid flow is considered to be laminar, incompressible and two-dimensional. Assuming a slight temperature difference, buoyancy effect is modeled by Boussinesq approximation. Thermophysical properties of the fluid are assumed to be constant; effects of radiation and viscous heat loss are excluded in the energy equation.

\subsection{Governing Equations}

Governing equations included equations of mass conservation, momentum, energy conservation, and rate of entropy generated. Conservative and dimensionless form of governing equations is as follows:

$$
\begin{aligned}
& \frac{\partial \mathrm{U}}{\partial \mathrm{X}}+\frac{\partial \mathrm{V}}{\partial \mathrm{Y}}=0 \\
& \mathrm{U} \frac{\partial \mathrm{U}}{\partial \mathrm{X}}+\mathrm{V} \frac{\partial \mathrm{U}}{\partial \mathrm{Y}}=-\frac{\partial \mathrm{P}}{\partial \mathrm{X}}+\operatorname{Pr} \times\left[\frac{\partial^{2} \mathrm{U}}{\partial \mathrm{X}^{2}}+\frac{\partial^{2} \mathrm{U}}{\partial \mathrm{Y}^{2}}\right] \\
& \mathrm{U} \frac{\partial \mathrm{V}}{\partial \mathrm{X}}+\mathrm{V} \frac{\partial \mathrm{V}}{\partial \mathrm{Y}}=-\frac{\partial \mathrm{P}}{\partial \mathrm{Y}}+\operatorname{Pr} \times\left[\frac{\partial^{2} \mathrm{~V}}{\partial \mathrm{X}^{2}}+\frac{\partial^{2} \mathrm{~V}}{\partial \mathrm{Y}^{2}}\right]+R a \cdot \operatorname{Pr} \cdot \theta \\
& \mathrm{U} \frac{\partial \theta}{\partial \mathrm{X}}+\mathrm{V} \frac{\partial \theta}{\partial \mathrm{Y}}=\frac{\partial^{2} \theta}{\partial \mathrm{X}^{2}}+\frac{\partial^{2} \theta}{\partial \mathrm{Y}^{2}} \\
& \mathrm{~N}_{\mathrm{s}}=\frac{1}{\left(\theta+T_{\infty}^{*}\right)^{2}}\left[\left(\frac{\partial \theta}{\partial \mathrm{X}}\right)^{2}+\left(\frac{\partial \theta}{\partial \mathrm{Y}}\right)^{2}\right]+ \\
& \frac{\mathrm{EcPr}}{\left(\theta+T_{\infty}^{*}\right)}\left[2\left\{\left(\frac{\partial \mathrm{U}}{\partial \mathrm{X}}\right)^{2}+\left(\frac{\partial \mathrm{V}}{\partial \mathrm{Y}}\right)^{2}\right\}+\left(\frac{\partial \mathrm{U}}{\partial \mathrm{X}}+\frac{\partial V}{\partial \mathrm{Y}}\right)^{2}\right]
\end{aligned}
$$

Governing equations are discretized by finite volume method and solved by using SIMPLER algorithm. A steady $50 \times 50$ grid network us used to store velocities and scalar variables. Moreover, Power Law approximation is used for the relationship between convectionpenetration terms. Finally, the linear algebraic equations are solved by inverse matrices method. 


\section{RESULTS AND DISCAUSIONS}

\subsection{Validation}

In order to validate computational code, the results were compared with work by Mukhopadhyay (2010) and it was observed that good agreement was made between the results under the same conditions. The result of this comparison has presented in Figure 2.

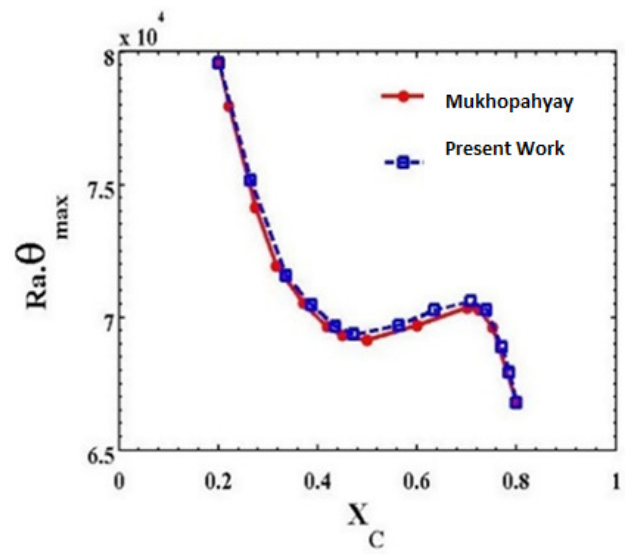

Fig. 2 Comparison of current results with work by Mukhopadhyay (2010)

To investigation of mesh independency of the network, dimensionless maximum temperature value was calculated in a special case for three different gridding. The results of these calculations have presented in Table 1.

Table 1 Mesh independency investigation of the network

\begin{tabular}{ccc}
\hline Change in $R a . \theta_{\max }$ & Ra. $\theta_{\max }$ & Network size \\
\hline \hline- & $0.81 \times 10^{5}$ & $25 \times 25$ \\
0.19 & $1 \times 10^{5}$ & $50 \times 50$ \\
0.001 & $1.001 \times 10^{5}$ & $100 \times 100$ \\
\hline
\end{tabular}

\subsection{Results}

\subsubsection{Effect of Rayleigh Number Change on Flow Field, Temperature and Entropy}

Figure 3 shows variations in streamlines and isotherm lines by changing Rayleigh number. In this case, heaters have equal length ratios (right heater to left heater) and power ratio (right heater to left heater) $\left(\varepsilon_{\mathrm{r}}=\mathrm{q}_{\mathrm{r}}=1\right)$, as well as equal distance between heaters and side walls. Pattern of streamlines shows that, viscosity is partially dominated by buoyancy force and buoyancy flow appears weak in low Rayleigh numbers $\left(\mathrm{Ra}=10^{3}\right.$ and $\mathrm{Ra}=10^{4}$ ). In this case, isotherms are smooth curves and practically the upper half of the enclosure is not influenced by heat sources. The pattern of isotherms reveals similarity of conductivity caused by heaters. As the Rayleigh number increases, vortex nucleus moves upward due to formation of a stronger rotational force and an increase in buoyancy force. By increasing the Rayleigh number due to formation of a stronger temperature gradient, isotherms deviate from pure conduction, and fluid movement causes hot air to pass through the cold walls. When $\mathrm{Ra}=10^{6}$, isotherms are thinner than other states, which is due to the increased energy transfer. In all states shown in Figure 3, the heaters have identical length and power ratios and boundary conditions of the lateral walls are symmetrical; therefore, similar heat dissipation occurs in the lateral cold walls and surface temperature of heaters is expected to become equal.
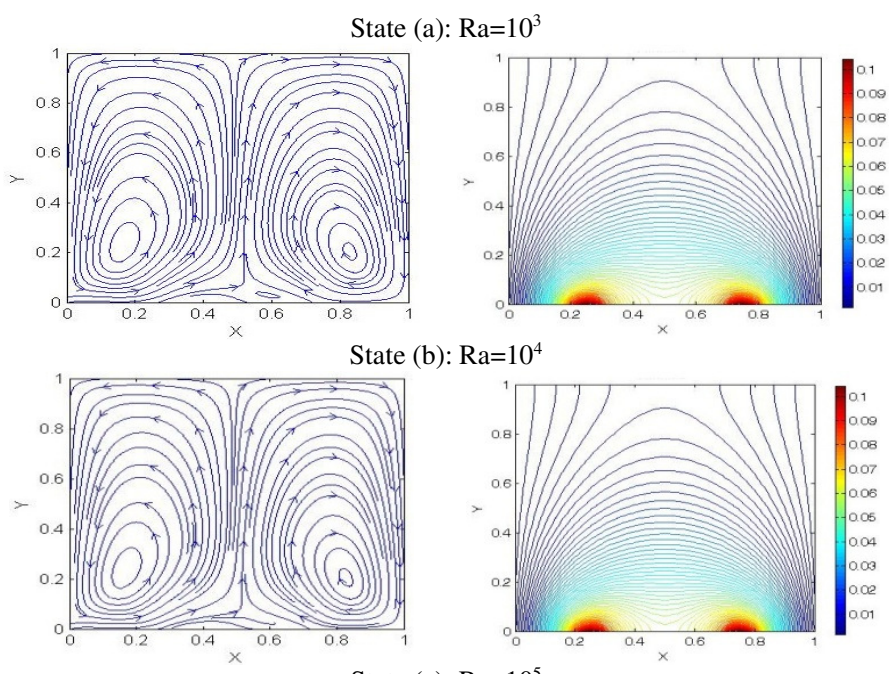

State (c): $\mathrm{Ra}=10^{5}$
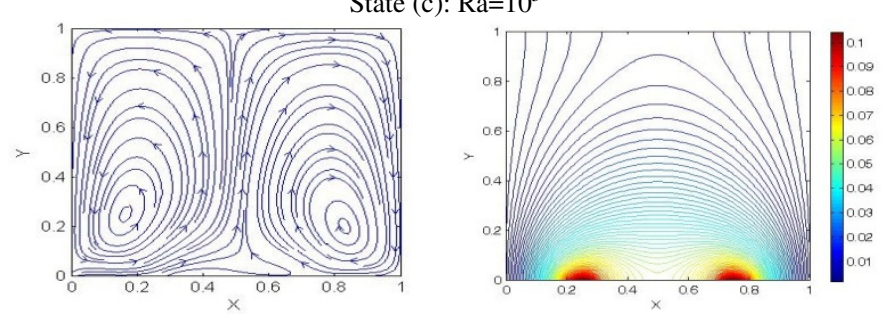

State (d): $\mathrm{Ra}=10^{6}$
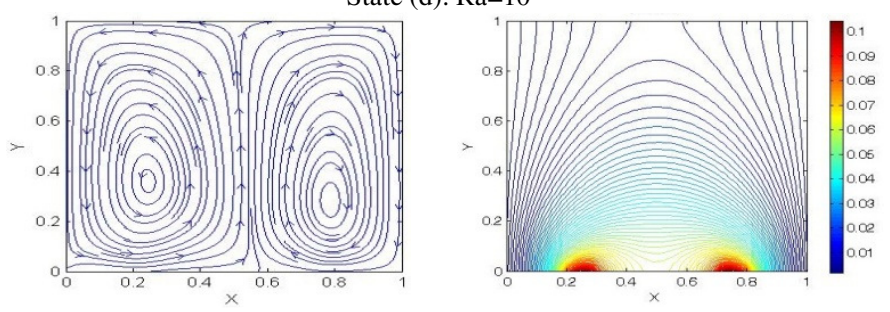

Fig. 3 Variations in streamline (left column) and isotherms (right column) by changing the Rayleigh number

Figure 4 shows variations in entropy generation contours caused by fluid friction (FFI) and conductive heat transfer (HTI) by changing the Rayleigh number. These contours are plotted using normalized values. These values are obtained by dividing entropy caused by conductive heat transfer $\left(\mathrm{N}_{\mathrm{s}, \text { conduction }}\right)$ or fluid friction $\left(\mathrm{N}_{\mathrm{s} \text {,viscous }}\right)$ by maximum entropy generated $\left(\mathrm{N}_{\mathrm{s}, \max }\right)$. These contours show that maximum entropy is generated near the heaters due to irreversibility caused by heat transfer (HTI). In this study, convection heat transfer is due to two temperature differences. These two temperature differences, which cause internal and external irreversibility due to heat transfer in the system, are:

1. The difference in temperature (convection heat transfer) between heaters and the fluid inside the enclosure.

2. The difference in temperature (convection heat transfer) between the hot fluid and the cold walls of the enclosure.

The first temperature difference causes internal irreversibility, and the second temperature difference causes external irreversibility due to heat transfer. Convective entropy generation diagram shows that HTI value is very small near the upper wall due to the lack of heat transfer in these areas. Increase in the Rayleigh number increases temperature difference and convection heat transfer between the heater and the fluid inside the enclosure; thus, as the Rayleigh number increases, conductive entropy generation rate increases. Diagrams of entropy generation resulting from fluid friction show that irreversibility caused by fluid friction is higher near the walls due to formation of the boundary layer and velocity gradient. As the Rayleigh number increases, fluid flow and thickness of the hydrodynamic boundary layer formed on the walls 
increases; thus FFI value increases. In all states examined in Figure 4, magnitude of the entropy generated by heat transfer is greater than the entropy generated by fluid friction. In other words, irreversibility is largely due to heat transfer. Hence, Bejan number, which represents the relative importance of irreversibility of heat transfer to total irreversibility, can be considered close to 1 for all states studied. This is consistent with work by Sivasankaran (2011), which found that entropy generation caused by fluid friction is several orders of magnitude smaller than the entropy generated by heat transfer. Figure 5 shows contours of variations in total entropy by changing the Rayleigh number. This case using 2500 computational nodes requires about 50 to 55 minutes of CPU-time by Pentium Dual-Core $2.6 \mathrm{GHz}, 4$ GB RAM computer.
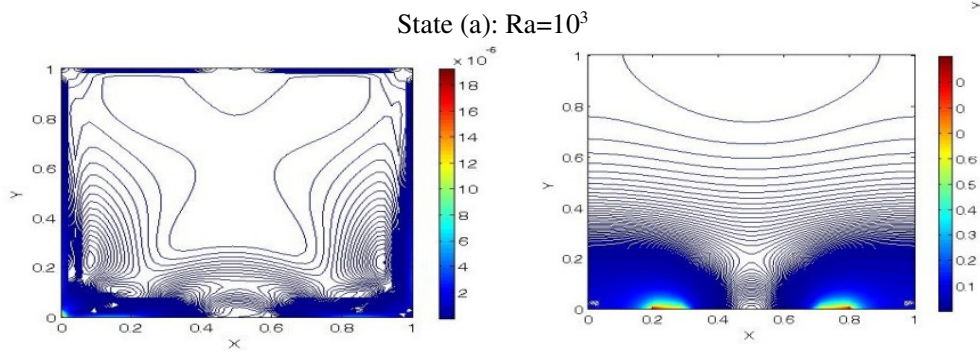

State (b): $\mathrm{Ra}=10^{4}$
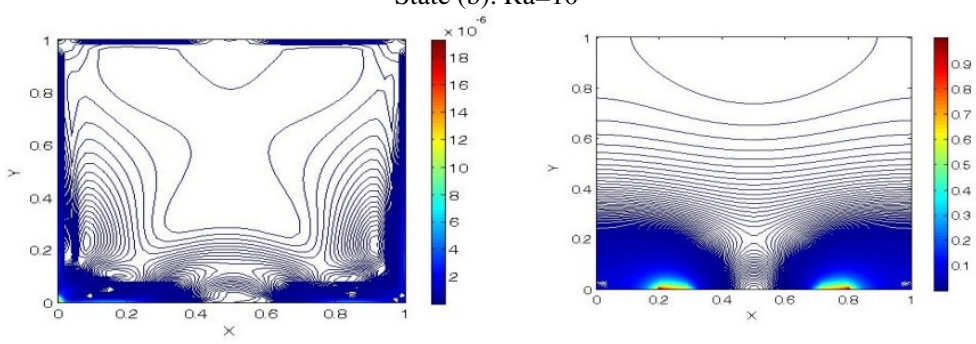

State (c): $\mathrm{Ra}=10^{5}$
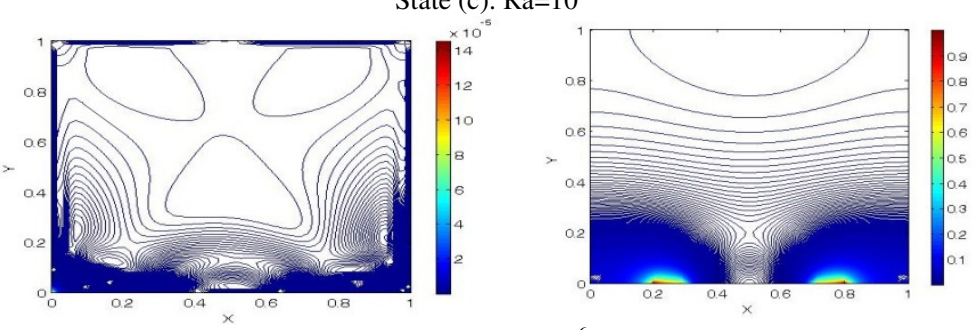

State (d): $\mathrm{Ra}=10^{6}$
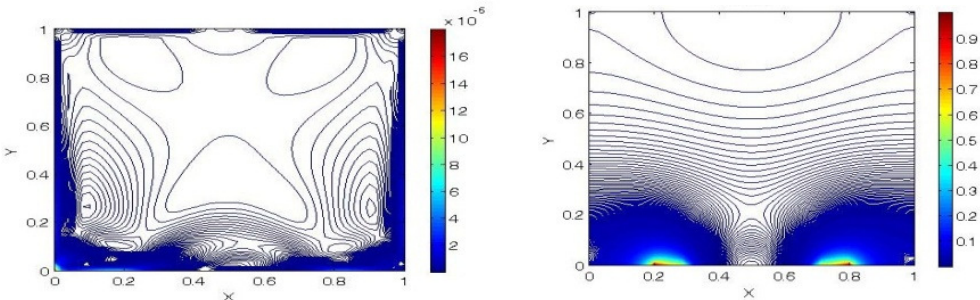

Fig. 4 Variations in entropy generated by fluid friction (left column) and conductive heat transfer (right column) by changing the Rayleigh number

As shown in Figure 5, as the Rayleigh number increases, total entropy generation increases in the enclosure due to simultaneous increase in entropy generation due to fluid friction and convection heat transfer.
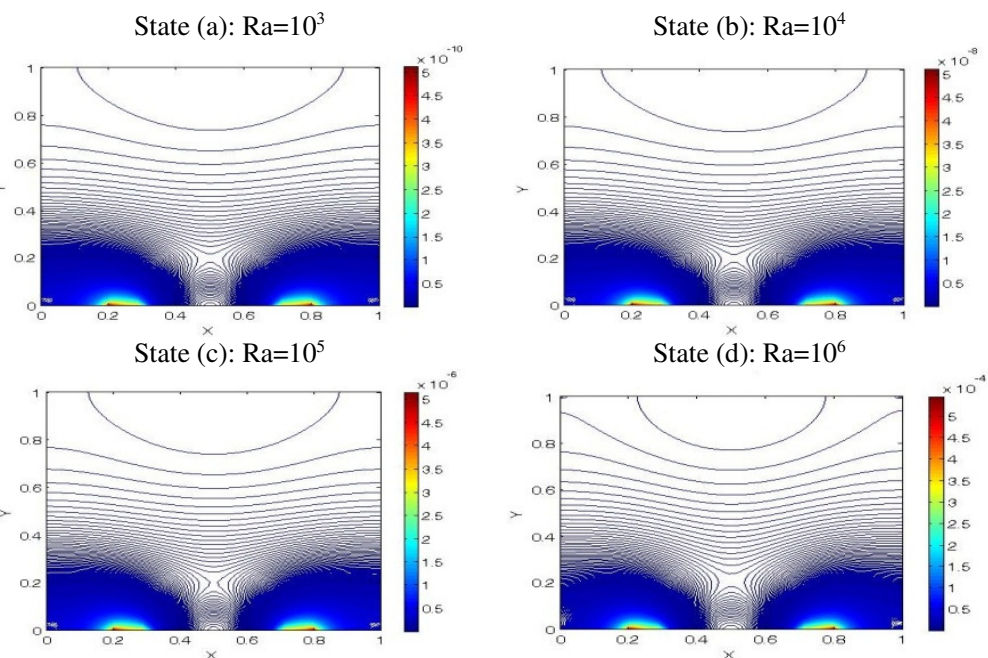

Fig. 5 Variations in total entropy by changing the Rayleigh number

\subsubsection{Effect of Change in Heater Power Ratio on Flow Field, Temperature and Entropy Generation}

Given that value of thermal energy applied to the enclosure is constant and its value depends on the size and power of the heaters; although total heat applied to the enclosure is constant, values of dimensionless ratios of the length and power of the heaters are changed to evaluate effect of these dimensionless parameters on flow and temperature fields. In different states, this study calculates the Rayleigh number so that total heat applied to the system is equivalent to a state where, $\varepsilon_{\mathrm{r}}=\mathrm{q}_{\mathrm{r}}=1$ and $\mathrm{Ra}=10^{6}$. For this purpose, the Rayleigh number is obtained by $\mathrm{Ra}=\left(2 \times 10^{6}\right) /\left(1+\varepsilon_{\mathrm{r}} \times \mathrm{q}_{\mathrm{r}}\right)$ in cases where the ratio of length or power of the heaters is changed. In cases where power of heaters is not equal, the role of convection from places with higher flux density and its dissipation through thermal conductivity from the side of the source is weaker. In other words, the role of lateral walls in relation to heat dissipation is deviation from the symmetry $\mathrm{q}_{\mathrm{r}}=1$ in the state where power of heaters is not equal. Figure 6 shows the effect of unequal power of heaters on flow and temperature fields. In all states associated with this figure, heaters have equal length $\left(\varepsilon_{\mathrm{r}}=1\right)$. In the state where $\mathrm{q}_{\mathrm{r}}=0.25$, power of the right heater is $25 \%$ of the left heater; in other words, the heat applied from the right heater is about $20 \%$ of total heat applied to the enclosure. In this state, dominant vortex is formed on top of the right heater and is distributed throughout the enclosure. This environment is the dominant environment in heat dissipation and leads to more heat dissipation from the right wall compared with the left wall.

For states where $\mathrm{q}_{\mathrm{r}}>1$, the heat provided by the right heater is greater than the left heater; this results in an increase in contribution of the left wall to total thermal dissipation and decrease in the average temperature of the left heater compared with the right heater. This can also be deduced from Figure 6. Therefore, the increase in $\mathrm{q}_{\mathrm{r}}$ reduces maximum temperature of the left heater, while maximum temperature of the right heater increases. On the other hand, the presence of a dominant vortex on the right heater indicates higher heat dissipation from surface of this heater compared to the left heater in states where $\mathrm{q}_{\mathrm{r}}<1$. 
State (a): $\mathrm{q}_{\mathrm{r}}=0.25$
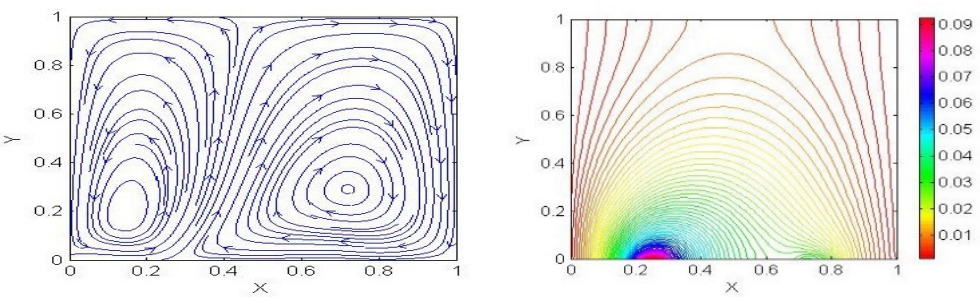

State (b): $\mathrm{q}_{\mathrm{r}}=0.75$
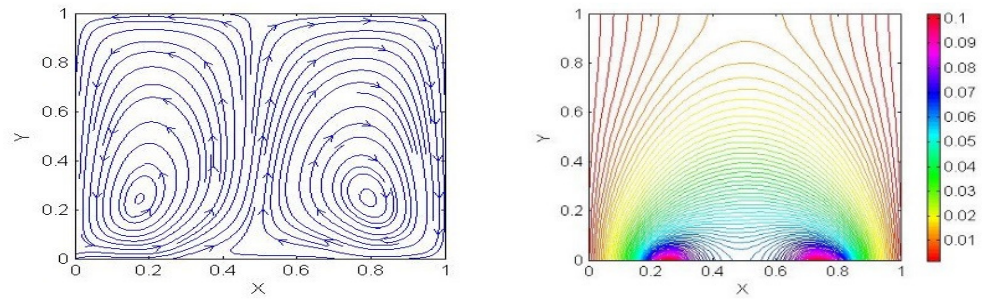

State (c): $\mathrm{q}_{\mathrm{r}}=1.25$
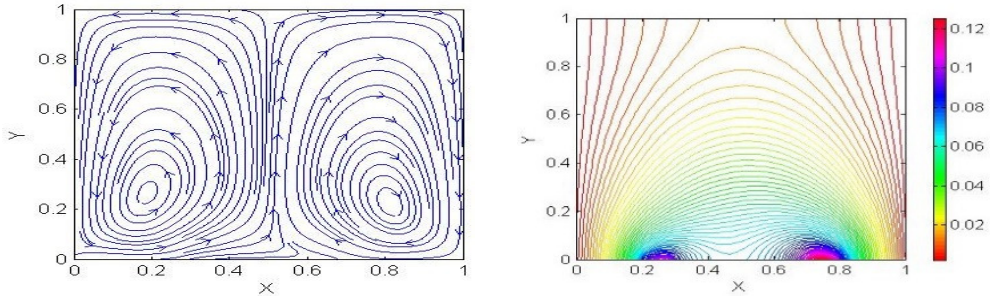

State (d): $\mathrm{q}_{\mathrm{r}}=1.75$
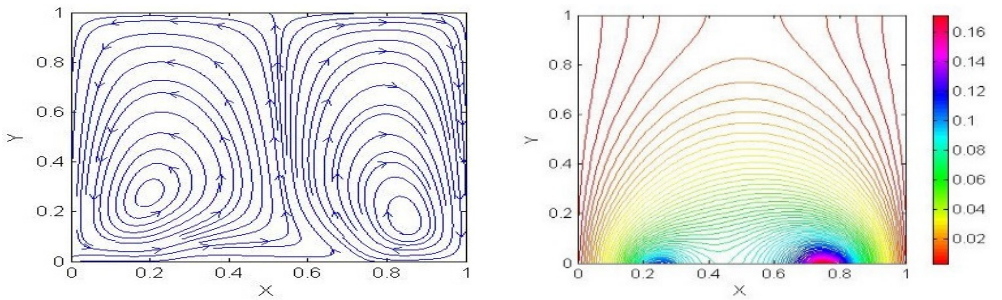

Fig. 6 Variations in streamlines (left column) and isotherms (right column) by changing flux ratio of heaters

Therefore, the right wall has a greater contribution in total heat dissipation for all states where $\mathrm{q}_{\mathrm{r}}<1$, resulting in a lower average temperature of the right heater than the left heater. It is also expected that maximum temperature has the same trend as the average temperature.

Figure 7 shows variations in surface temperature of the heaters by changing their power ratios for different values of the heater length ratios. The states examined in this figure show that increase in power ratios of the heaters reduces maximum temperature of the left heater for each length ratio of the heaters but maximum temperature of the right heater increases. Moreover, comparison of different states of this figure shows that optimal power ratio is related to the state where peak temperature of both heaters is identical in the state where length of the heater is unequal. This ensures minimum value of maximum temperature in the enclosure.

Figure 8 shows variations in total entropy generated and the entropy generated by fluid friction by changing power ratio of the heaters. These contours show that entropy generation is lower in areas near the center of the enclosure, while irreversibility is higher in areas close to the heaters and lateral walls. FFI contours indicate that magnitude of the entropy generated by fluid friction is higher in areas close to the stronger heater due to presence of higher velocity gradient caused by formation of a stronger floating flow near the heater with higher power.
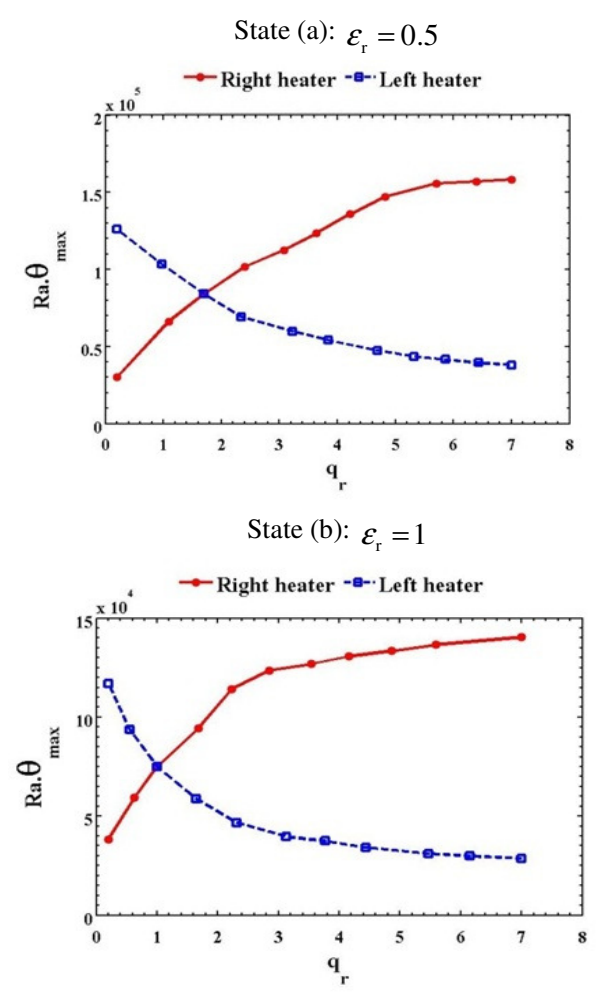

State (c): $\varepsilon_{\mathrm{r}}=1.5$

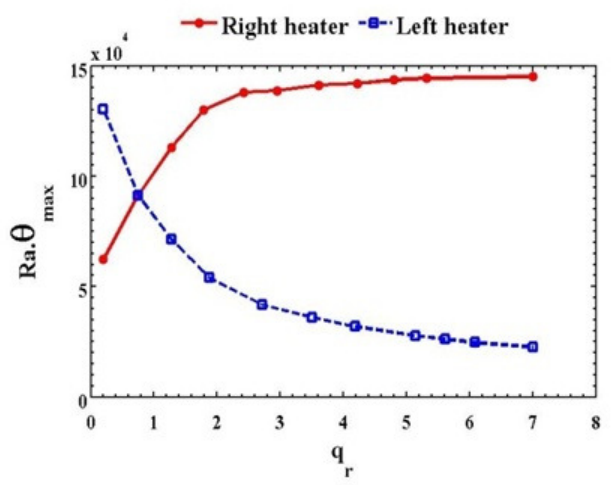

Fig. 7 Variations in temperature of the left and right heater by changing their power ratio

Total entropy contours indicate that entropy generation is greater in areas near the stronger heater due to formation of a higher temperature gradient between the heater and its adjacent fluid. Moreover, the increase in power ratio of the heaters reduces the generated entropy for states where $\mathrm{q}_{\mathrm{r}}<1$ (states a and $\mathrm{b}$ ), while the increase in power ratio of heaters increases total entropy for states where $q_{r}>1$ (states $c$ and $d$ ). Figure 9 shows variations in total entropy generation rate and surface temperature of the heaters by varying their power ratios for a given value of heater length ratio. Comparison of the states examined in this figure shows that increase in power ratios of the heaters first reduces, minimizes and then increases entropy generation rate for any length ratio of the heaters. In asymmetric states of the heater length ratios ( $\varepsilon_{\mathrm{r}}=0.5$ and $\varepsilon_{\mathrm{r}}=1.5$ ), there is a significant difference in the entropy rate generated relative to the unit state $\left(\varepsilon_{\mathrm{r}}=1\right)$ for a given power ratio. In these states, higher entropy generation close to a stronger heater causes an increase in total entropy generation rate compared to the unit state. This case using 2500 computational nodes requires about 60 to 65 minutes of CPU-time 
State (a): $\mathrm{q}_{\mathrm{r}}=0.25$
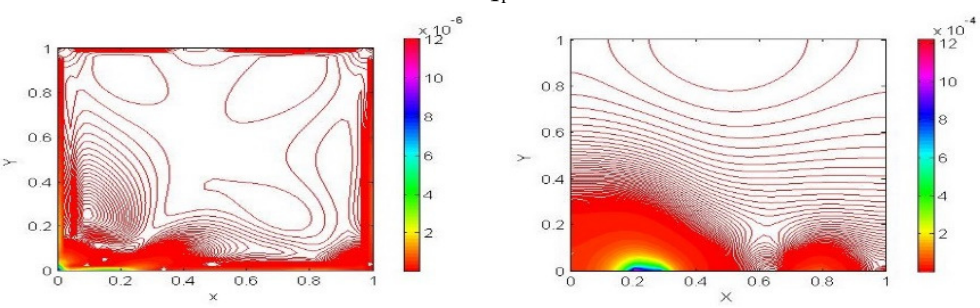

State (b): $\mathrm{q}_{\mathrm{r}}=0.75$
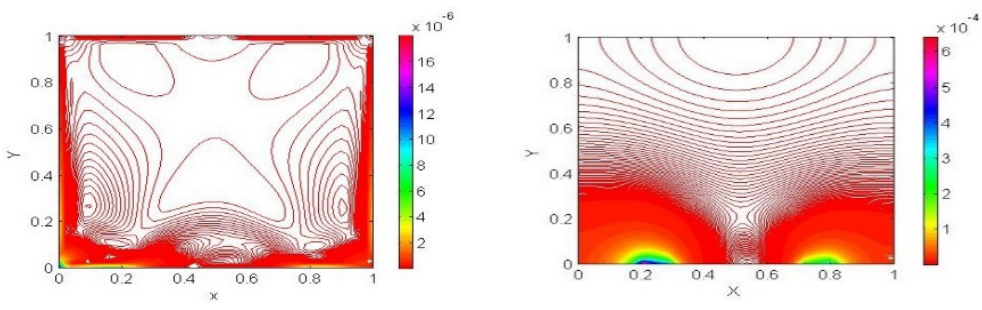

State (c): $\mathrm{q}_{\mathrm{r}}=1.25$
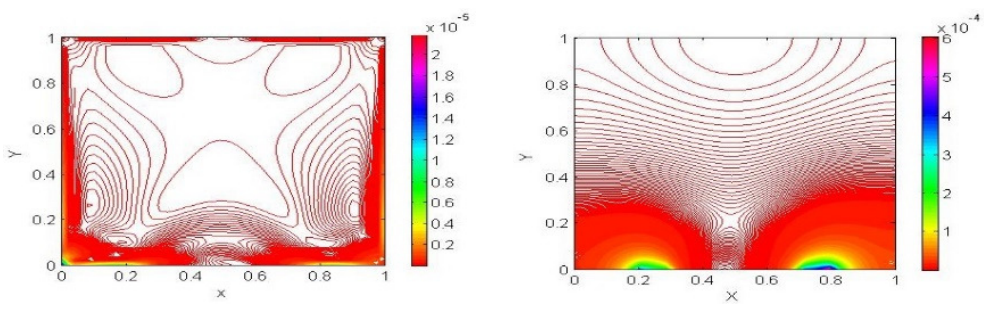

State (d): $\mathrm{q}_{\mathrm{r}}=1.75$
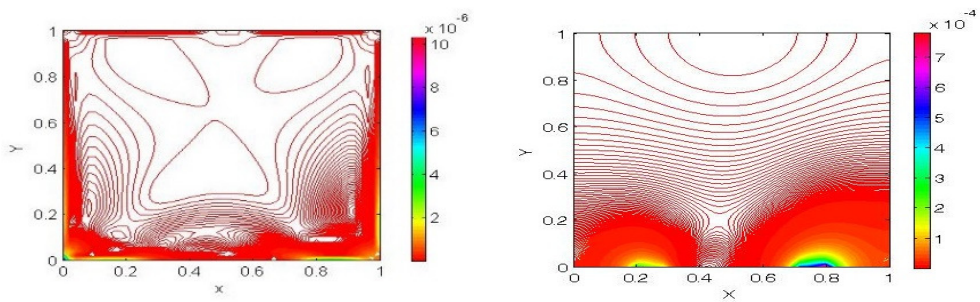

Fig. 8 Variations in total entropy generation (right column) and entropy generation caused by fluid friction (left column) by changing power ratio of heaters

Another valuable result which can be derived from this figure is that the power ratio that generates equal maximum temperature on the heaters also generates minimum entropy for any length ratio of the heaters. Therefore, there is an optimal arrangement for several discrete thermal sources with an unequal length or power, for which peak temperature and entropy generated are minimized.

\section{CONCLUSIONS}

This study tended to optimize dimensionless parameters of Rayleigh number, length ratio and power of heat sources. The results presented as diagrams of streamlines, isotherms, heater surface temperatures and entropy maps showed that, in low Rayleigh numbers, the dominant heat transfer was conduction by fluid layers; by increasing Rayleigh number, the heat transfer mechanism was changed to convection and effects of conduction were eliminated. In areas where temperature gradient was higher and isotherms were more compact, entropy generation caused by heat transfer (HTI) was higher. The non-slip boundary condition resulted in formation of a hydrodynamic boundary layer on the walls; thus, velocity gradient and entropy generation due to fluid friction were higher near the walls. As the Rayleigh number increased, the heat generated and fluid motion increased in the enclosure, followed by an increase in reversibility due to fluid friction (FFI) and heat transfer (HTI).
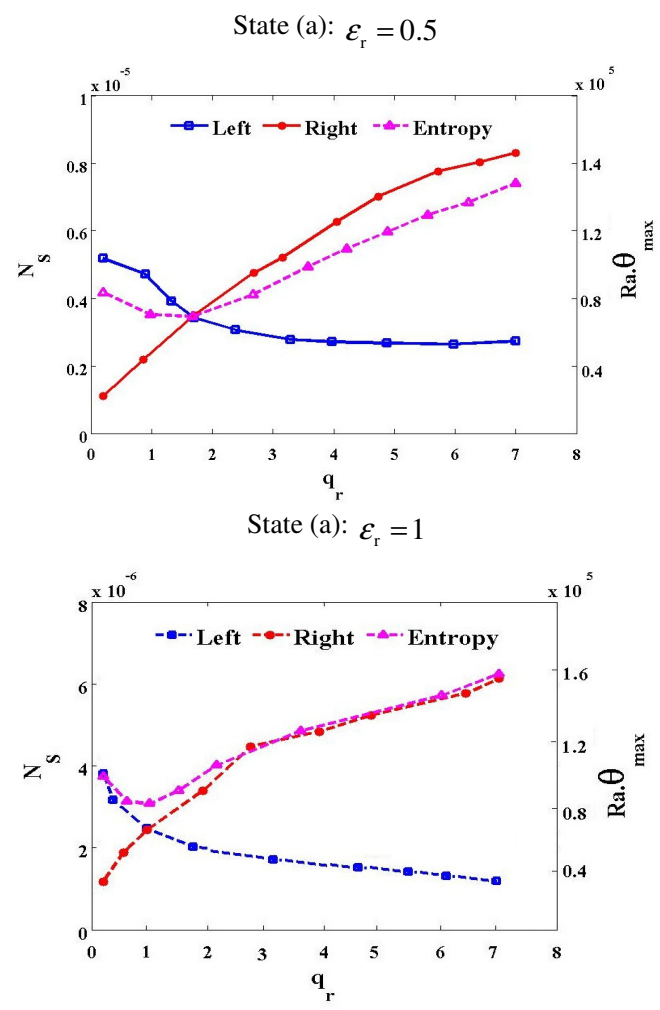

State (b): $\varepsilon_{\mathrm{r}}=1.5$

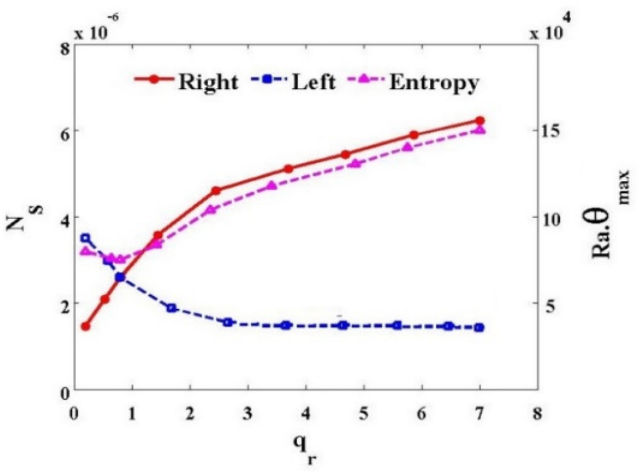

Fig. 9 Variations in heater temperature and total entropy generation by changing power ratio of heaters

In this study, a major part of entropy generation was related to reversibility due to heat transfer, and a small part of total entropy generated was related to fluid friction. For all cases examined, the arrangement which generated minimum peak temperature of the heater generated minimum irreversibility. In cases examined, minimum entropy generation rate was related to the state where the heaters had equal power ratio.

\section{NOMENCLATURE}

EC Eckert number $\left(U_{\infty}^{2} / C_{P}\left(T_{S}-T_{\infty}\right)\right)$

$P \quad$ dimensionless pressure $\left(p / \rho u_{\infty}^{2}\right)$

$\operatorname{Pr} \quad$ Prandtl number $(v / \alpha)$

$q_{r} \quad$ heat flux of right heater to left heater

$R a \quad$ Rayleigh number $\left(g \beta\left(T_{S}-T_{\infty}\right) H^{3} / v \alpha\right)$

$T^{*} \quad$ dimensionless temperature $(\mathrm{K})$ 


$$
\begin{array}{ll}
U, V & \text { dimensionless velocity in } \mathrm{x} \text { and y direction }\left(u / u_{\infty}\right) \\
X, Y & \text { dimensionless coordinate }(x / H, y / H)
\end{array}
$$

\section{Greek Symbols}

$\mathcal{E}_{r} \quad$ length of right heater to left heater

$\theta \quad$ dimensionless temperature $\left(\frac{T-T_{\infty}}{T-T_{S}}\right)$

\section{REFERENCES}

Chadwick, M.L., 2008, "Natural Convection from Two-dimensional Discrete Heat Sources in a Rectangular Enclosure," Int. J. Heat Mass Transfer. 34(7), 1679-1693.

https://doi.org/10.1016/0017-9310(91)90145-5

Deng, Q., and Tang, H., 2002, "A combined temperature scale for analyzing natural convection in rectangular enclosures with discrete wall heat sources," Int. J. Heat Mass Transfer, 45, 3437-3446. https://doi.org/10.1016/S0017-9310(02)00060-1

De Vahl, D., 1983, "Natural Convection of Air in a Square Cavity :a Benchmark Solution," Int. J. Numer. Meth. Fluids, 3, 249-264. https://doi.org/10.1002/fld.1650030305-1

Ezzatabadipour, M., Zahedib, H., and Keshtkar, M.M., 2017, "Fluid Flow Simulation in a Random Elliptical Porous Medium by Using the Llattice Boltzmann Method," Journal of Applied Mechanics and Technical Physics, 58 (3), 379- 385.

$\underline{\text { https://link.springer.com/article/10.1134/S0021894417030014 }}$

Ganjalikhan Nassb, S.A., Bahrami, A., Keshtkar, M.M., Safaripour M.H., 2014, "Comparison Between Two Methods for Enhancing Heat Transfer in Separated Flows Over Backward Facing Step - Second Law Analysis," IJST, Transactions of Mechanical Engineering, 38, 9-23. https://doi.org/ 10.22099/ijstm.2014.19237

Gdhaidh, F.A., 2014, "Numerical Investigation of Conjugate Natural Convection Heat Transfer from Discrete Heat Sources in Rectangular Enclosure," Proceedings of the World Congress on Engineering, Vol II, WCE, July, London, U.K.

$\underline{\text { https://bradscholars.brad.ac.uk/handle/10454/8021 }}$

Keshtkar, M.M., and Gandjalikhan Nassab, S.A., 2009, "Theoretical Analysis of Porous Radiant Burners under 2-D Radiation Field Using Discrete Ordinates Method," Journal of Quantitative Spectroscopy \& Radiative Transfer, 110, 825-835.

https://doi.org/10.1016/j.jqsrt.2009.04.007

Keshtkar, M.M., Gandjalikhan Nassab, S.A., and Nasr M. R. J., 2009, "Heat Transfer Characteristics of a Cylindrical Porous Radiant Air Heater under the Influence of Two-dimensional Axisymmetric Radiative Field," Proc. IMechE Part A: J. Power and Energy, 223, 913923.

https://doi.org/10.1243/09576509JPE788

Keshtkar, M.M., 2016, "Numerical Simulation of Fluid Flow in Random Granular Porous Media Using Lattice Boltzmann Method," Int. J. Advanced Design and Manufacturing Technology, 9(2), 31-40. https://admt.iaumajlesi.ac.ir/

Keshtkar, M.M., and Amiri, B., 2017, "Numerical Simulation of Radiative-Conductive Heat Transfer in an Enclosure with an Isotherm Obstacle," Heat Transfer Engineering, 38, 125-136.

https://doi.org/10.1080/01457632.2017.1280293
Keshtkar, M.M., and Ghazanfari, M., 2017, "Numerical Investigation of Fluid Flow and Heat Transfer Inside a 2D Enclosure with Three Hot Obstacles on the Ramp under the Influence of a Magnetic Field," Engineering, Technology \& Applied Science Research, 7(3), 16471657.

http://etasr.com/index.php/ETASR/article/view/1115/522

Mukhopadhyay, A., 2010, "Analysis of entropy generation due to natural convection in square enclosures with multiple discrete heat sources," International Communications in Heat and Mass Transfer, 37, 867-872.

https://doi.org/10.1016/j.icheatmasstransfer.2010.05.007

Nithyadevi, N., Kandaswamy, P. and Lee J., 2007, "Natural convection in a rectangular cavity with partially active side walls," Int. J. Heat Mass Transfer, 50, 4688-4697.

https://doi.org/10.1016/j.ijheatmasstransfer.2007.03.050

Onyango O.M., 2008, "Enhancement of Natural Convection Heat Transfer in a Square Enclosure with Localized Heating from Below," Int. J. Heat Mass Transfer, 60, 3958-3966. https://doi.org/10.1016/j.csite.2018.01.008

Rahimi, A.R., Kasaeipoor, A., Hasani Malekshah, E., and Kolsi L., 2017, "Natural Convection Analysis by Entropy Generation and Heatline Visualization using Lattice Boltzmann Method in Nanofluid Filled Cavity Included with Internal Heaters- Empirical ThermoPhysical Properties," International Journal of Mechanical Sciences, 133, 199-216.

https://doi.org/10.1016/j.ijmecsci.2017.08.044

Rahimi, A.R., Kasaeipoor, A., Hasani, Malekshah, E., and Kolsi L., 2017, "Experimental and Numerical Study on Heat Transfer Performance of Three-dimensional Natural Convection in an Enclosure Filled with DWCNTs-Water Nanofluid," Powder Technology, 322, 340-352.

https://doi.org/10.1016/j.powtec.2017.09.008

Salari, M., Hasani, Malekshah, E., Hasani, Malekshah, M., Alavi, M., and Hajihashemi, R., 2017, "3D Numerical Analysis of Natural Convection and Entropy Generation within Tilted Rectangular Enclosures Filled with Stratified Fluids of MWCNTs/Water Nanofluid and Air," Journal of the Taiwan Institute of Chemical Engineers, 80, 624-638.

https://doi.org/10.1016/j.jtice.2017.08.041

Saitoh, T. and Hirose, K., 1989, "High-Accuracy Benchmark Solutions to Natural Convection in a Square Cavity," Comput Mech., 4, 417-427. https://doi.org/10.1007/BF00293047

Sivasankaran, S., Do, Y., and Sankar, M., 2011, "Effect of Discrete Heating on Natural Convection in a Rectangular Porous Enclosure," Transport in porous media, 86, 261-281.

https://link.springer.com/article/10.1007/s11242-010-9620-x

Salari, M., Hasani, Malekshah, E., and M.H. Malekshah, 2017," Natural Convection in a Rectangular Enclosure Filled by Two Immiscible Fluids of Air and Al2O3-Water Nanofluid Heated Partially from Side Walls," Alexandria Engineering Journal, (In press)

https://doi.org/10.1016/j.aej.2017.07.004

Yeong, H. and Jung, M., 2000, “A Numerical Study on Threedimensional Conjugate Heat Transfer of Natural Convection and Conduction in a Differentially Heated Cubic Enclosure with a Heat Generating Cubic Conducting Body," Int. J. Heat Mass Transfer, 43, 4229-4248.

https://doi.org/10.1016/S0017-9310(00)00063-6 